\title{
Optical Dialysis Adequacy Monitoring: Small Uremic Toxins and Contribution to UV-Absorbance Studied by HPLC
}

\author{
Kai Lauri1 ${ }^{1}$ Jürgen Arund ${ }^{1}$, Jana Holmar ${ }^{1}$, Risto Tanner ${ }^{1,2}$, \\ Merike Luman ${ }^{3}$ and Ivo Fridolin 1 \\ ${ }^{1}$ Department of Biomedical Engineering, Technomedicum, Tallinn University of Technology, \\ ${ }^{2}$ Laboratory of Chemical Physics, National Institute of Chemical Physics and Biophysics, \\ ${ }^{3}$ Department of Dialysis and Nephrology, North Estonia Medical Centre,
}

Estonia

\section{Introduction}

Uremic toxicity is described as a clinical picture resulting from impaired renal elimination and accumulation of uremic toxins in the body. Uremic toxins can be classified as small water-soluble compounds, middle molecules and protein-bound compounds. A long list of possible uremic toxins has been identified in recent decades. Under normal conditions these compounds are excreted by healthy kidneys. If kidney function fails, waste products accumulate in the blood and in the body. Dialysis treatment replaces some kidney functions through diffusion (waste removal) and ultrafiltration of fluid across a semi-permeable membrane, which is a thin layer of material with holes or pores of various sizes. A deeper understanding about the accumulation and removal mechanisms of the retained solutes during care of renal insufficiency is needed (Eloot, 2008; Eloot, 2007). This understanding would be especially informative with respect to predicting the mode of action of uremic toxins and their specific role in complications associated with dialysis or ureamia, but also with cardiovascular disease and inflammation (Vanholder, et al., 2008; Vanholder, 2003). The methods contributing to the identification, characterisation and evaluation of uremic retention solutes could be assessed in order to ensure dialysis adequacy and quality (Vanholder, 2005). The choice of the correct concentration of potential uremic toxins is still an unresolved issue (Vanholder, 2003). In everyday clinical practice, uremic components are not examined due to the measurement of most uremic components using the available laboratory methods being difficult and complex. A number of standard biochemical techniques are used in clinical laboratories, but there is no universal methodology. In addition, some chromatographic methods have been developed which explore uremic retention solutes.

Dialysis efficiency and quality has been an important issue accompanied by optimisation and the best outcome of the treatment of chronic end stage renal disease for many years. In connection to this, online monitoring of the dialysis dose has been suggested as an effective way of improving treatment quality. The concept of online monitoring is based on the realtime measurement of chemical signals coming from the patient. This enables the early 
recognition of signs of intolerance and allows for early intervention. Despite the first online monitors of dialysis doses being available today, it is essential to monitor the patient's condition during the dialysis session and, if necessary, change some conditions of dialysis treatment (e.g. time) more specifically considering uremic toxins with various characteristics and elimination profiles.

The aim of this chapter is to describe and assess performance of optical dialysis adequacy monitoring technique related to the removal of uremic retention solutes during dialysis. The assessment is based on the high performance liquid chromatography (HPLC) profiles of the serum and the spent dialysate connected to the origin of the cumulative and integrated UV absorbance arising from the contribution of uremic retention solutes - chromophores, among them probably several uremic toxins.

\section{Dialysis adequacy and online monitoring}

Dialysis adequacy means providing a sufficient amount of dialysis treatment to maintain a uremic patient in the best condition. The goal of qualitatively treated dialysis is to prevent complications due to uremic toxicity.

Some recent studies are reviewed suggesting that uremic toxins are involved in the progression of renal failure and are at least partially removed by hemodialysis (Lesaffer, 2000; Eloot, 2008; Vanholder, 2003). The efficiency of a dialysis session has been estimated through concentration of uremic toxins measured before and after the dialysis session. A classic marker of the dialysis dose and adequacy is a small molecule urea.

Two methods are generally used to assess dialysis adequacy: URR and Kt/V. The urea reduction ratio (URR) is based on tests of blood urea, by measuring the levels before and after treatment to show how much has been removed. The Kt/ $\mathrm{V}$ is mathematically related to the URR and can be derived from it (NKF-DOQI, 2006). In a simplified model of urea removal from a fixed volume with no urea generation, $\mathrm{Kt} / \mathrm{V}$ is related to URR as follows:

$$
\mathrm{Kt} / \mathrm{V}=-\ln (1-\mathrm{URR})
$$

In general, this method of dialysis adequacy is based on pre- and post-dialysis measurements of urea concentration. Online methods are considered to be more accurate than methods based on pre- and post-dialysis urea concentrations and have been found to be better suited to clinical routines. Online monitoring of the dialysis dose has been suggested as a valuable tool in ensuring adequate dialysis prescription (Locatelli, 2005).

\section{Optical dialysis online monitoring}

Two optical techniques have made progress toward clinical use over the past ten years namely the ultraviolet (UV) absorbance and near infrared (NIR) techniques. The experimental results indicate very good correlation between UV absorbance and several small solutes such as urea, creatinine and uric acid in the spent dialysate and in the blood for every individual treatment at a fixed wavelength of $285 \mathrm{~nm}$ (Fridolin, 2002). Good correlation between UV absorbance and a small removed waste solute such as urea enables the determination of $\mathrm{Kt} / \mathrm{V}$ for urea (Fridolin, 2003). The NIR method can measure urea directly using signal processing of the raw NIR spectra (Cho, 2008; Eddy, 2001; Eddy, 2003).

To implement NIR technology as a simple and robust sensor based on Fourier transform near-infrared (FT-NIR) spectrometer or acousto optical tunable filter (AOFT) based 
spectrometer is far more complicated because of the underlying optical principle, in which interference or diffraction is utilised (Jensen, 2002; Cho, 2008). The UV method is more straightforward and is not as demanding considering the source and detector characteristics and other technological modules. The UV method has recently been commercialised as a monitoring tool for dialysis dose in terms of the urea-based parameters, Kt/V and URR. Validation studies of the system have shown that the results with UV technology are indistinguishable from blood based Kt/V (Castellarnau, 2010).

An optical dialysis online monitoring system utilising UV absorbance has been developed for the continuous monitoring of the toxins eliminated by the spent dialysate. This system represents a spectrophotometer connected to the fluid outlet of the dialysis machine. All spent dialysate passes through a specially designed optical cuvette. The transmitted light intensity of the spent dialysate is measured. All substances - chromophores - absorbing the UV-radiation at a particular wavelength, give the total online UV absorbance curve. The schematic clinical set-up of online monitoring experiments is shown in Figure 1.

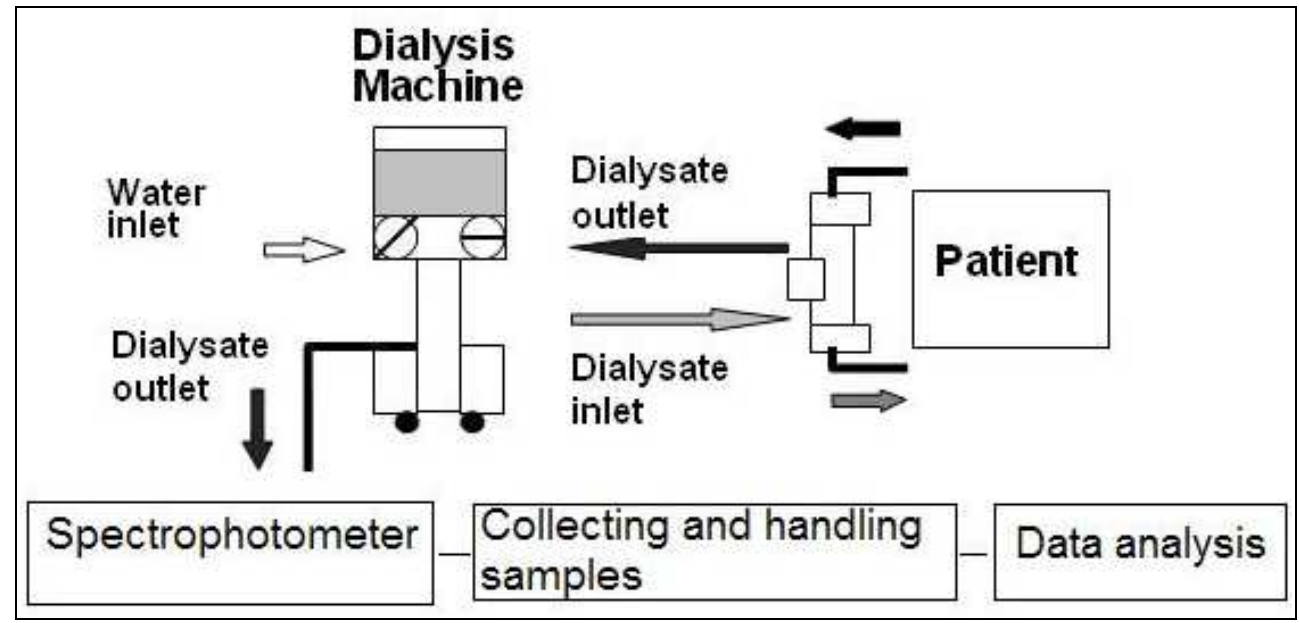

Fig. 1. Clinical experimental set-up and sample analysis

The obtained UV absorbance values are processed and presented on the computer screen by a PC incorporated in the spectrophotometer using special software. The absorbance A of a solution, obtained by the spectrophotometer using the pure dialysate as the reference solution, is determined as:

$$
A=\log \frac{I_{r}}{I_{r+s}}
$$

where $I_{r}$ is the intensity of transmitted light through the reference solution (pure dialysate) and $I_{r+s}$ is the summated intensity of transmitted light through the reference solution containing the solutions under study (pure dialysate + waste products from the blood). The absorbance is measured in arbitrary units (a.u.). A sampling frequency at two samples per minute is usually sufficient, but can be much higher (e.g. if a more detailed curve is desired or noise reduction is necessary). 
The examples of two online signals measured at $280 \mathrm{~nm}$ from two different dialysis treatments are shown in Figure 2. The UV absorbance is higher at the beginning of treatment because of the high concentration of metabolic waste products in the body fluids. When the waste products are removed from the blood the UV absorbance decreases during the dialysis session. The times at which the blood and dialysate samples were collected are also shown in this figure. Blood samples were drawn before the start of dialysis treatment $\left(\mathrm{B}_{\text {start }}\right)$ and immediately after the treatment $\left(B_{\text {end }}\right)$. Dialysate samples were taken 10 minutes after the start of the dialysis session ( $\left.D_{\text {start }}\right)$ and at the end of the treatment ( $\left.D_{\text {end }}\right)$ (210 or 240 minutes).

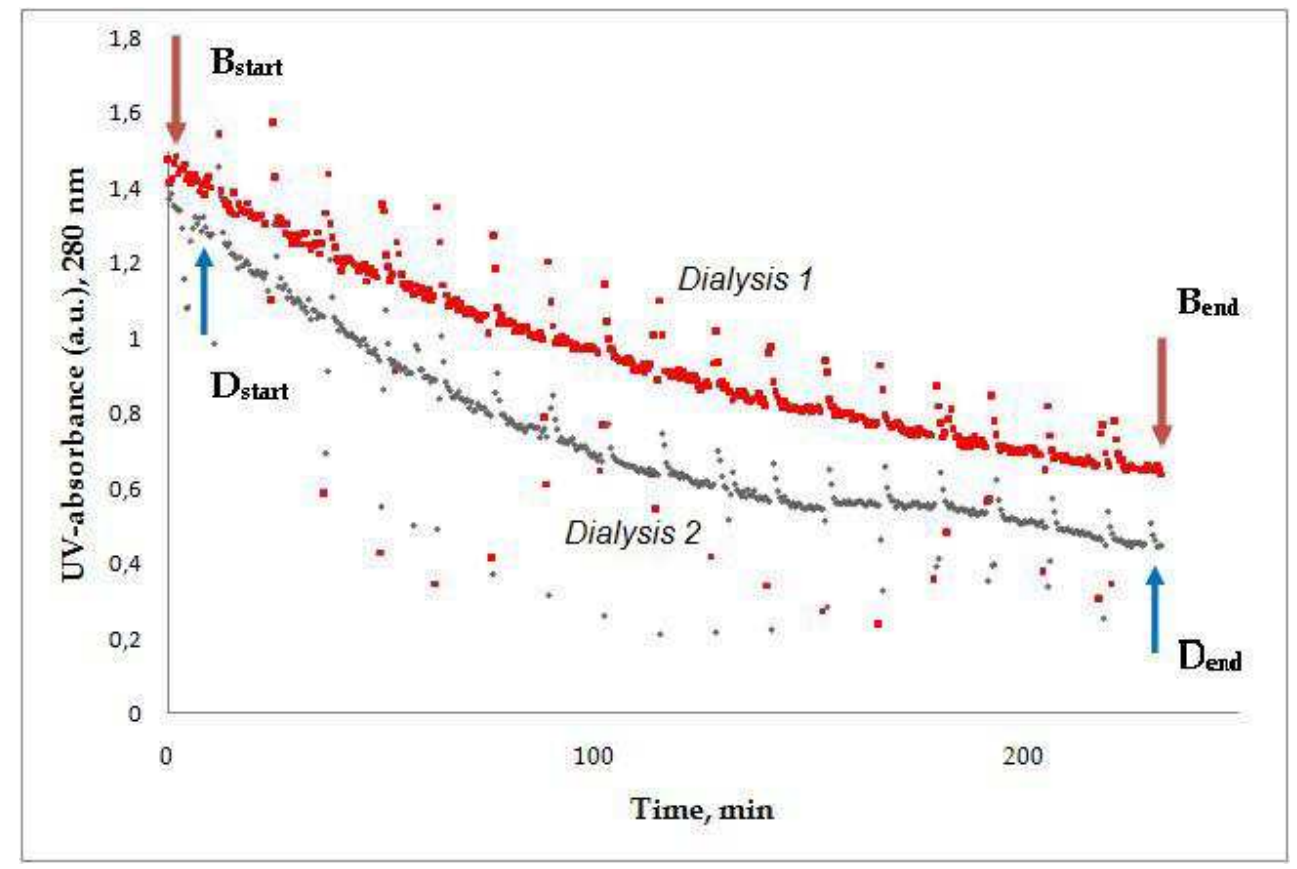

Fig. 2. Example of two different online UV absorbance measurements (at $280 \mathrm{~nm}$ ). Time points when the samples were taken for later analysis are as follows: $D_{\text {start }}$ - dialysate sample collected 10 minutes after start of hemodialysis, $\mathrm{D}_{\text {end }}$ - dialysate sample collected at the end of hemodialysis, $\mathrm{B}_{\text {start }}$ - blood sample collected before dialysis session, $\mathrm{B}_{\text {end }}$ - blood sample collected at the end of hemodialysis

This figure presents a classic picture of UV absorbance signals obtained by the optical online dialysis dose monitor, which can be used for estimation of uremic solutes removal.

As seen from Figure 2, the UV absorbance curves are somewhat different. The exponential decrease of the UV absorbance curve represents the elimination rate of the all UV-absorbing compounds - chromophores - which varies from patient to patient and from treatment to treatment. Good correlation between the UV absorbance measured in the spent dialysate and the concentration of several uremic retention solutes, both in the spent dialysate and in the blood of the dialysis patients, has been previously shown (Fridolin, 2002). For these reasons the origin of the cumulative and integrated UV absorbance arising from the contribution of uremic retention solutes, among them probably several uremic toxins, should be investigated. 


\section{HPLC as an analysis tool for biological samples}

High performance liquid chromatography (HPLC) is a technique of analytical chemistry which can separate and identify the components of a mixture of different chemical compounds in liquid solution. The reversed-phase HPLC technique is the most commonly used form of HPLC. This method is recommended as a sensitive, accurate and reproducible tool for qualitative and quantitative analysis of aqueous samples (Vanholder, 2001). Furthermore, the use of ambient temperature in reversed-phase columns makes it possible to investigate the many non-volatile or thermally unstable compounds commonly found in biological samples. The principle involved in HPLC testing enables the separation of compounds in a mixture more efficiently and faster than that of traditional column chromatography.

In general, the HPLC system consists of two essential components - a stationary phase and a mobile phase. The stationary phase is a column packed with small solid sorbent particles and where the separation of different compounds takes place. The mobile phase is a flowing liquid (solvent) that transports the compounds from the sample through the stationary phase. Thus, the compounds of the mixture travel at different rates due to their relative affinities with the solvent and stationary phase. Separation of compounds in the stationary phase occurs with slight differences in chemical properties, such as chemical polarity and size of non-polar groups.

Figure 3 is a simple block diagram illustrating the main components of a modern HPLC. A system consists of several units: pumping, sample-injection, separation (column), detection and data-processing.

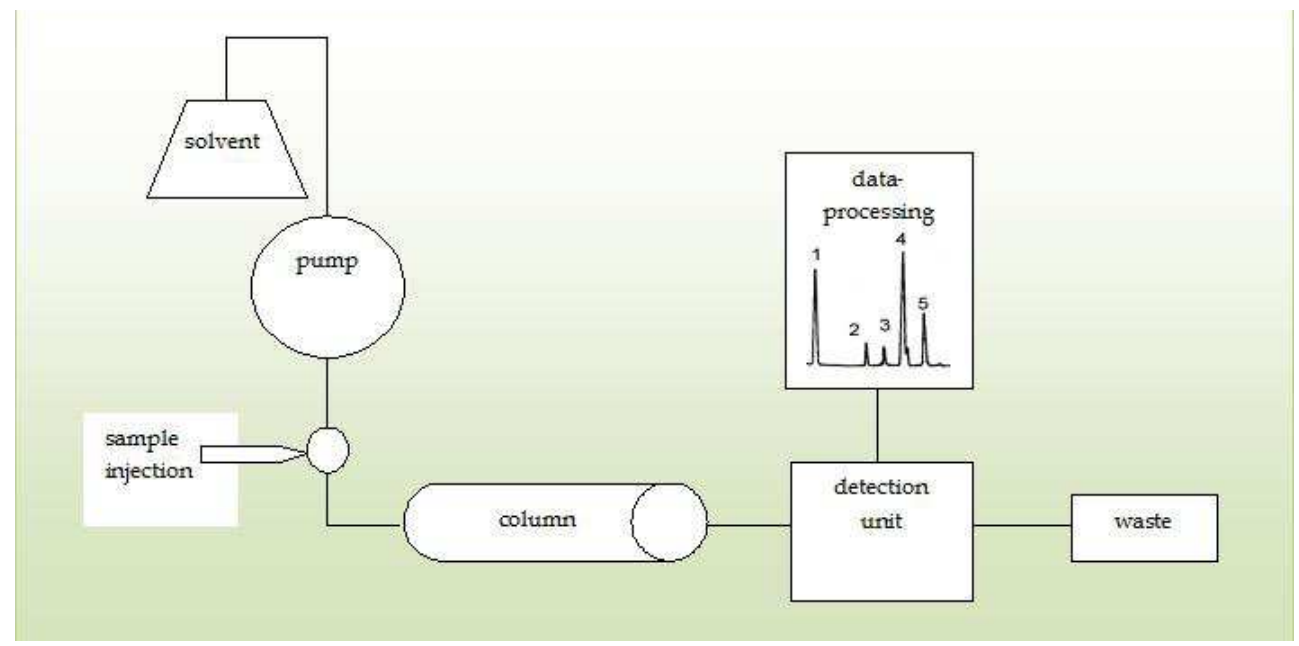

Fig. 3. Schematic reversed-phase HPLC method principle

The results of chromatographic analysis are known as chromatograms, where the signal intensity from the detector is recorded on the time axis. For HPLC there are several different detection methods; the most popular are optical. An ultraviolet-visual light (UV-VIS) absorption detector is the main optical detection method. This detector is effective in the detection of components with an absorption wavelength of $400 \mathrm{~nm}$ or less in the ultraviolet 
region. There are three types of UV-VIS detectors: a fixed wavelength detector; a variable wavelength detector; and a diode array detector (DAD). However, in modern HPLC systems DAD is commonly used, which in addition to the UV-VIS signal offers the ability to produce an absorption spectrum for every time slice during the chromatogram. A DAD detects the absorption in the UV to VIS region. Using the DAD, absorption on a large number of wavelengths can be measured simultaneously. This enables us to select the best wavelengths for actual analysis. Additionally, one of the advantages of DAD is that it allows us to detect sufficiently pure peaks. Often the peak shape itself does not reveal that it actually corresponds to two or even more components. In such a case, absorbance rationing on several wavelengths is particularly helpful in deciding whether the peak represents a single compound or a composite peak. However, compared with a UV-VIS detector, the DAD is susceptible to changes, such as lamp fluctuations, and noise is large because the amount of light is small.

For those compounds which fluoresce or which exhibit an appropriate fluorescence due to derivatisation, a fluorescence detector is the most sensitive of the existing modern HPLC detectors. Its sensitivity is 10-1000 times higher than that of the UV detector for strong UVabsorbing materials. Fluorescence detectors are very specific and selective compared to other optical detectors. A universal detector, but the least sensitive for non-ionic compound monitoring, is the refractive index detector. To estimate oxidisable and reducible compounds, the most suitable is the electrochemical detector.

Reversed-phase HPLC has been found to be useful in the analysis of uremic biofluids. A number of authors have reported the application of the HPLC method in the analysis of dialysate and serum samples already in several decades ago (Schoots, 1989; Schoots, 1982). Different detectors have been used. Some naturally fluorescent compounds have been separated and identified with a fluorescence detector, in both serum and haemodialysate (Niwa, 1993; Barnett, 1985; Swan, 1983). A liquid chromatographic method including a UV detector for detection of UV-absorbing solutes in uremic serum has been developed (Schoots, 1985; Senftleber, 1976; Knudson 1978). A fixed wavelength at $254 \mathrm{~nm}$ was mainly used in these studies. Additionally, several types of chromatographic methods, such as gas chromatography mass spectrometry (GC-MS), for the detection of uremic toxins has also been developed (Niwa, 1997; Niwa, 2009). Mass spectrometry (MS) has been applied to the identification and quantification of uremic toxins and uremia-associated modified proteins (Niwa, 2009). However, GC-MS cannot analyse highly polar, thermally labile and highmolecular weight compounds and usually requires sample preparation, such as extraction or derivation to make non-volatile compounds thermally stable and volatile. Compared with GC-MS, liquid chromatography mass spectrometry (LC-MS) can separate and identify highly polar, thermally labile or high-molecular weight mixture compounds and does not require derivation; sample preparation is also simple. Newly developed LC-MS techniques have been successfully applied to uremic toxin research with the discovery of novel uremic toxins that range from low-molecular weight solutes to small-molecule proteins (Niwa, 2011). This new analytical method is available today, opening up new horizons for uremic toxin identification detected earlier as unidentified HPLC peaks.

\section{HPLC study of UV absorbance profiles}

In order to explain the origin and potential of the online UV absorbance dialysis dose monitoring method, the HPLC method of analysing UV absorbance profiles was developed. 
Ten uremic patients - three females and seven males (mean age $62.6 \pm 18.6$ years) participated in the study. All of the patients were investigated over a course of 30 hemodialysis treatments. Three different polysulphone dialysers were used (Fresenius Medical Care, Germany): a low flux membrane dialyser F8 HPS (N=14), a low flux membrane dialyser F10 HPS (N=3) and a high flux membrane dialyser FX $80(\mathrm{~N}=11)$. The elimination of toxins by the different types of semi-permeable membranes was also estimated.

Blood samples were drawn before the start of dialysis treatment $\left(B_{\text {start }}\right)$ and immediately after the treatment $\left(B_{\text {end }}\right)$ (Figure 2$)$ using the slow flow/stop pump sampling technique. Blood was sampled into BD Vacutainer ${ }^{\circledR}$ Glass Serum Tube (red cup, Beckton Dickinson) and was allowed to clot. After centrifugation at 3000 r.p.m. the serum was ready for clinical chemistry analysis. Small molecules - creatinine (MW=113.12 Da), uric acid (MW=168.11 $\mathrm{Da})$ and urea (MW=60.06 Da) - were measured at the Clinical Chemistry Laboratory of the North Estonia Medical Centre using standardised methods (Hitachi 912 autoanalyser, Roche, Switzerland). The accuracy for creatinine was $5 \%$, for uric acid $2 \%$ and for urea $4 \%$.

For HPLC analysis the additional pre-treatment of the centrifugated serum was necessary to release the blood proteins. The serum samples were purified from proteins by centrifuging with two different Microcon centrifugal filters (Millipore, USA): a YM-3 with cut-off $3 \mathrm{kDa}$ and a YM-100 with cut-off $70 \mathrm{kDa}$. The aim of using different filters was to estimate the presence of different-sized molecules in the serum.

Dialysate samples were taken 10 minutes after the start of the dialysis session $\left(D_{\text {start }}\right)$ and immediately after the treatment ( $\mathrm{D}_{\text {end }}$ ) (210 or 240 minutes) (Figure 2$)$. Also, pure dialysate, used as the reference solution, was collected before each dialysis session, when the dialysis machine was being prepared and the conductivity was stable. For HPLC analysis, the dialysate samples were acidified with formic acid to $\mathrm{pH}$ 4.0. Sample sizes of $50 \mu \mathrm{L}$ or $100 \mu \mathrm{L}$ were used for chromatographic separation. Figure 4 presents a block diagram illustrating the collection and handling of blood and dialysate samples.

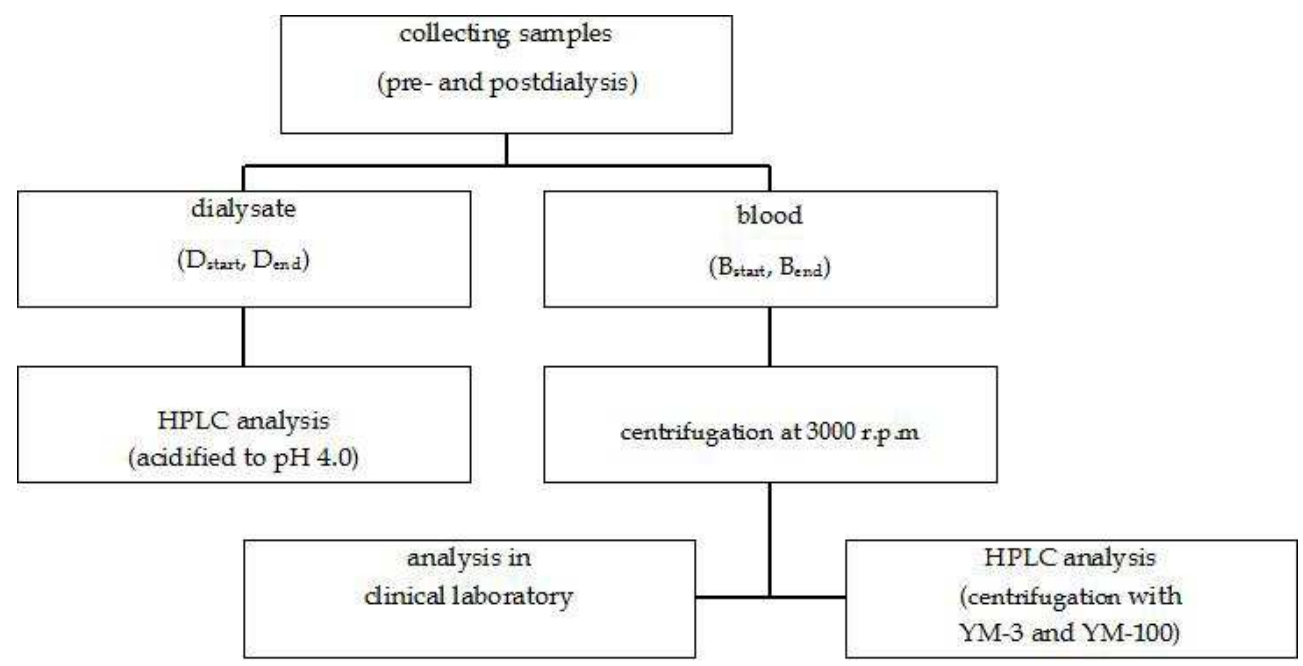

Fig. 4. Block diagram of blood and dialysate sample collection and handling 
The HPLC instrumentation and tools were as follows: a diode array spectrophotometric detector (DAD, Perkin Elmer, USA); a manual injector (Rheodyne, USA); and a Zorbax C8 4.6 x $250 \mathrm{~mm}$ column (Du Pont Instruments, USA) with security guard KJO-4282 (Phenomenex, USA). The eluent was mixed with $0.05 \mathrm{M}$ formic acid ( $\mathrm{pH} 4.0$ ), HPLC grade methanol and HPLC-S grade acetonitrile (Rathburn, Scotland), with a six-step gradient programme. The total flow rate of $1 \mathrm{~mL} / \mathrm{min}$ was used continuously and the column temperature was adjusted to $30^{\circ} \mathrm{C}$. The method followed has been described previously (Lauri, 2010). The chromatographic peaks were detected by the UV detector at wavelengths of 254 and $280 \mathrm{~nm}$. The data was processed by means of Turbochrom WS and Turboscan 200 software from Perkin Elmer.

The decrease of the uremic retention solutes was estimated as the reduction ratio (RR) and assessed as a percentage (\%). Thus, the RR of compounds was defined as a function of predialysis concentration $\left(\mathrm{C}_{\text {pre }}\right)$ and concentration at the end of hemodialysis $\left(C_{\text {post }}\right)$ and calculated as:

$$
R R=\frac{C_{\text {pre }}-C_{\text {post }}}{C_{\text {pre }}} 100 \%
$$

\subsection{Results}

The results from the studies obtained by the HPLC method in order to analyse the UV absorbance profiles of the serum and dialysate samples are presented below. The results are presented as mean \pm standard deviation (SD). A student's t-test was used to compare groups of values wherein $\mathrm{p}<0.05$ was considered significant. Pearson's correlation coefficient $(\mathrm{r})$ between the UV absorbance from HPLC and online monitoring versus concentration of the substances in the blood was investigated. Samples taken at times coinciding with the selftests or alarms of the dialysis machine were excluded ( 3 of 60 dialysate samples). Some sessions were excluded due to the technical failure of the spectrophotometer ( 3 of 30 sessions) and due to laboratory errors ( 3 of 30 sessions). The data analyses were performed in Microsoft Excel 2003 (for Windows).

The characteristic HPLC profiles of the spent dialysate at the start of the dialysis session $\left(D_{\text {start }}\right)$ and at the end $\left(D_{\text {end }}\right)$ are presented in Figure 5. When comparing the start and end values, the decrease in the height of the peaks (due to solute removal from the blood and into dialysate during dialysis) can be clearly seen.

As can be seen from this figure, a number of higher prevalent peaks can be observed from the HPLC profiles indicating the presence of chromophores, which are the main cause of cumulative and integrated UV absorbance. The highest peak on the HPLC profile is detected as uric acid causing a substantial amount of UV absorbance.

Figure 6 shows the HPLC chromatograms of the serum (filter YM-3 with cut-off $3 \mathrm{kDa}$ ) measured on wavelengths of $254 \mathrm{~nm}$ and $280 \mathrm{~nm}$. Identified HPLC peaks, such as creatinine, uric acid (the highest contribution), hypoxanthine, indoxyl sulphate and hippuric acid, are shown. Absorbing spectra of two unknown persistent peaks (P1 and P2) were identified at retention times (RT) of 15.46 and $15.82 \mathrm{~min}$.

Some chromophores, such as P2, creatinine, hypoxanthine and hippuric acid, give higher peaks on a wavelength of $254 \mathrm{~nm}$, while $\mathrm{P} 1$ and indoxyl sulphate are better identified on a wavelength of $280 \mathrm{~nm}$. This is due to the absorbing spectrum characteristics of the UV chromophores. This confirms the results obtained by the spectrophotometric analysis in this UV region. 


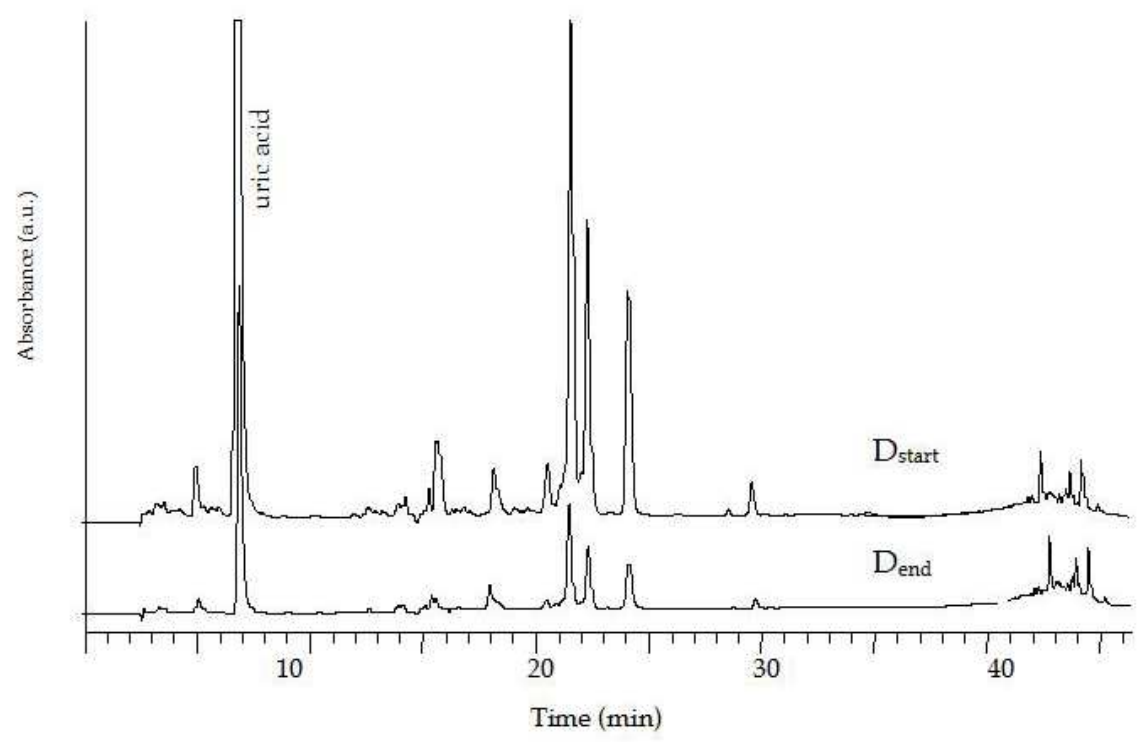

Fig. 5. Characteristic HPLC profiles of spent dialysate monitored on a wavelength of 280 $\mathrm{nm} ; \mathrm{D}_{\text {start }}$ - dialysate sample collected 10 minutes after start of hemodialysis, $\mathrm{D}_{\text {end }}$ - dialysate sample collected at the end of hemodialysis

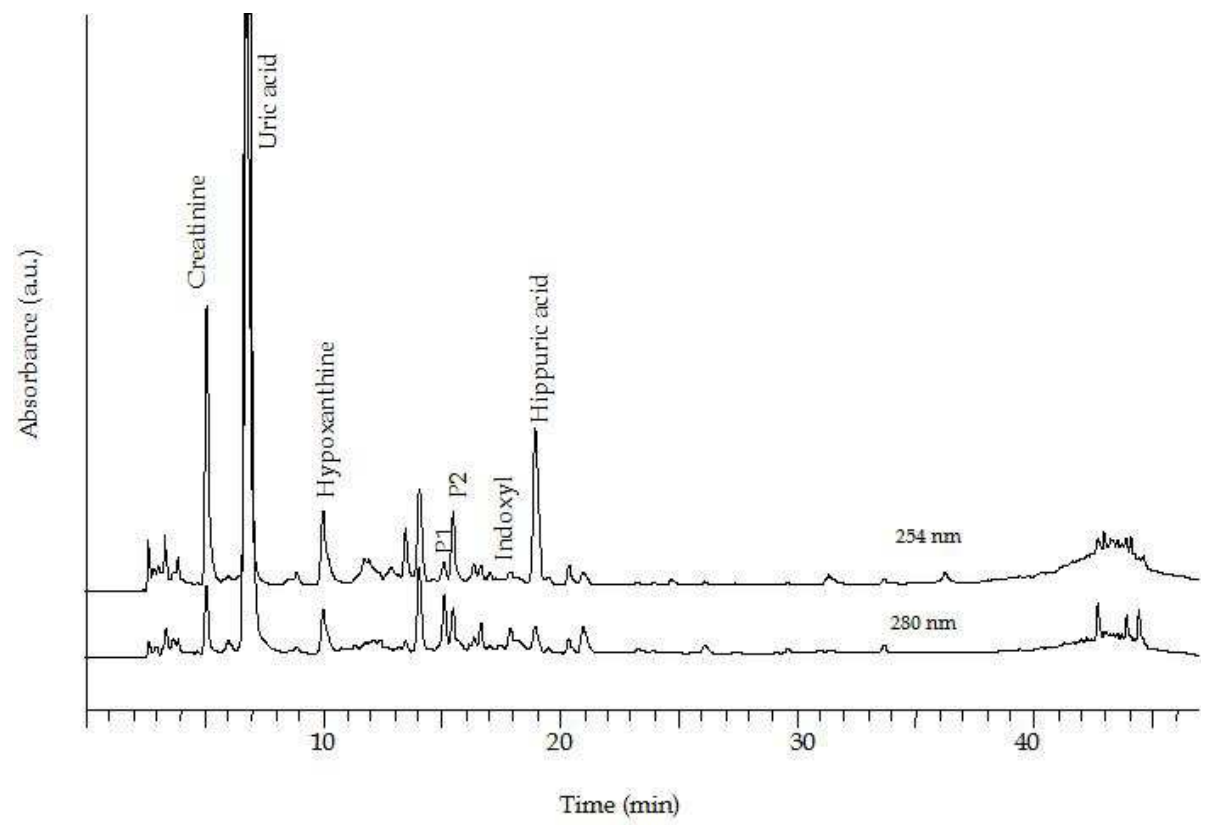

Fig. 6. Characteristic HPLC profiles of serum monitored on different wavelengths. Blood samples were collected before the dialysis session $\left(B_{\text {start }}\right)$ and monitored on wavelengths of $254 \mathrm{~nm}$ and $280 \mathrm{~nm}$. Detected HPLC peaks - uremic toxins - are presented. 
As seen from Figure 6, around ten peaks on the HPLC profiles can be selected forming the major part of the total HPLC signal. As a result of this, the total area of the ten main peaks (Top 10 area \%) was estimated. The comparison for Top 10 area \% in the serum and dialysate is presented in Figure 7. The contribution of ten main peaks forms approximately $80-90 \%$ of all HPLC peaks in both spent dialysate and serum. However, there is a difference in the number of peaks on different wavelengths. In comparison with the $254 \mathrm{~nm}$ wavelength, the Top 10 area \% on the $280 \mathrm{~nm}$ wavelength is higher. According to the HPLC profiles obtained (Figure 5 and 6), the largest contribution to Top 10 area \% on the $280 \mathrm{~nm}$ wavelength originates from the small water-soluble non-protein-bound uremic toxin uric acid. Additionally, there was no significant difference between the serum results filtered with different type of filter cut-off $(3 \mathrm{kDa}$ and $70 \mathrm{kDa})(\mathrm{p}<0.05)$.

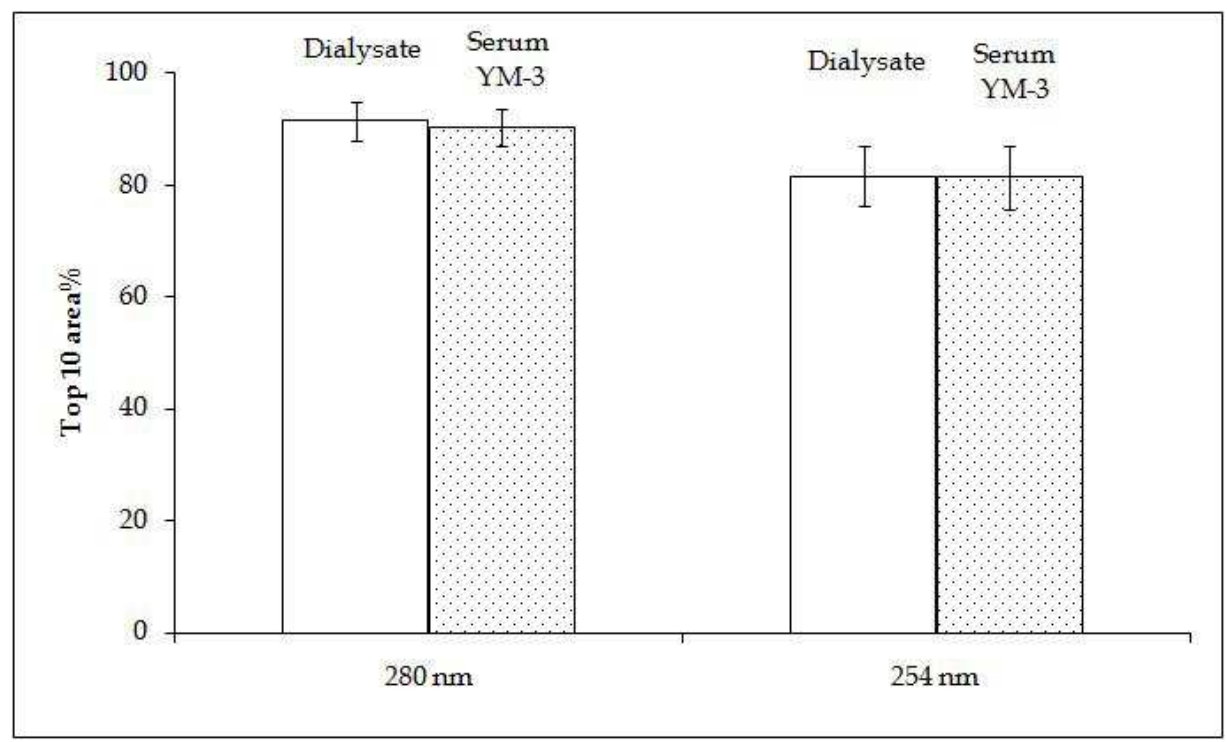

Fig. 7. Comparison of Top 10 area \% on wavelengths of $254 \mathrm{~nm}$ and $280 \mathrm{~nm}$ in serum (filter YM-3 with cut-off $3 \mathrm{kDa}$ ) and spent dialysate

The RR of the detected chromatographic solutes was estimated. Table 1 presents the comparison of the RR (\%) of solutes and all HPLC UV absorbance peaks on wavelengths of $254 \mathrm{~nm}$ and $280 \mathrm{~nm}$ in serum and for the online UV absorbance at $280 \mathrm{~nm}$ in the spent dialysate for different types of membranes: low flux (F8 HPS, F10 HPS) and high flux (FX80). There was no significant difference between the results for RR of low flux and high flux membranes $(\mathrm{p}<0.05)$.

Hippuric acid had the highest RR, while the small water-soluble compound hypoxanthine and protein-bound solute indoxyl sulphate had the lowest RR. The RR of all HPLC Ppeaks at $280 \mathrm{~nm}$ was similar to uric acid and urea and higher than the RR of online UV absorbance at $280 \mathrm{~nm}$ and creatinine. At the same time, the RR of all HPLC peaks at $254 \mathrm{~nm}$ and P2 were lower than uric acid and urea. Online UV absorbance at $280 \mathrm{~nm}$, creatinine and P2 were all removed in a statistically similar way $(\mathrm{p}<0.05)$ and had lower RR than uric acid, urea and all HPLC peaks at $280 \mathrm{~nm}$. 


\begin{tabular}{|c|c|c|}
\hline & \multicolumn{2}{|c|}{ RR (\%) } \\
\hline & Low flux & High flux \\
\hline Hippuric acid & $75.1 \pm 11.5(\mathrm{~N}=15)$ & $68.1 \pm 9.4(\mathrm{~N}=10)$ \\
\hline Uric acid & $67.7 \pm 8.5(\mathrm{~N}=15)$ & $65.6 \pm 6.7(\mathrm{~N}=15)$ \\
\hline Urea & $67.0 \pm 8.7(\mathrm{~N}=15)$ & $63.2 \pm 5.07(\mathrm{~N}=15)$ \\
\hline All HPLC peaks, $280 \mathrm{~nm}$ & $65.2 \pm 9.6(\mathrm{~N}=15)$ & $60.6 \pm 7.9(\mathrm{~N}=14)$ \\
\hline P1 & $62.1 \pm 13.0(\mathrm{~N}=12)$ & $61.0 \pm 5.3(\mathrm{~N}=7)$ \\
\hline All HPLC peaks, $254 \mathrm{~nm}$ & $60.2 \pm 12.5(\mathrm{~N}=14)$ & $57.2 \pm 7.7(\mathrm{~N}=13)$ \\
\hline P2 & $59.2 \pm 17.5(\mathrm{~N}=13)$ & $51.6 \pm 5.9(\mathrm{~N}=12)$ \\
\hline Online UV absorbance, $280 \mathrm{~nm}$ & $58.1 \pm 8.3(\mathrm{~N}=15)$ & $57.0 \pm 10.4(\mathrm{~N}=13)$ \\
\hline Creatinine & $58.2 \pm 7.7(\mathrm{~N}=15)$ & $56.6 \pm 5.4(\mathrm{~N}=15)$ \\
\hline Hypoxanthine & $42.6 \pm 16.0(\mathrm{~N}=10)$ & $46.1 \pm 18.5(\mathrm{~N}=8)$ \\
\hline Indoxyl sulphate & $42.1 \pm 18.0(\mathrm{~N}=13)$ & $47.8 \pm 14.0(\mathrm{~N}=12)$ \\
\hline
\end{tabular}

Table 1. RR (\%) of solutes and total area of all HPLC UV absorbance peaks on wavelengths of $254 \mathrm{~nm}$ and $280 \mathrm{~nm}$ in serum and RR of online UV absorbance at $280 \mathrm{~nm}$ in spent dialysate for different types of membranes

As the low flux and high flux membranes showed similar RR for every uremic solute, the results can be combined. Figure 8 present an illustrative comparison between the RR of serum uric acid, urea and creatinine measured in the clinical laboratory using standardised methods, all HPLC peaks measured on two different wavelengths (254 and $280 \mathrm{~nm}$ ) and dialysate online UV absorbance measurement (280 nm).

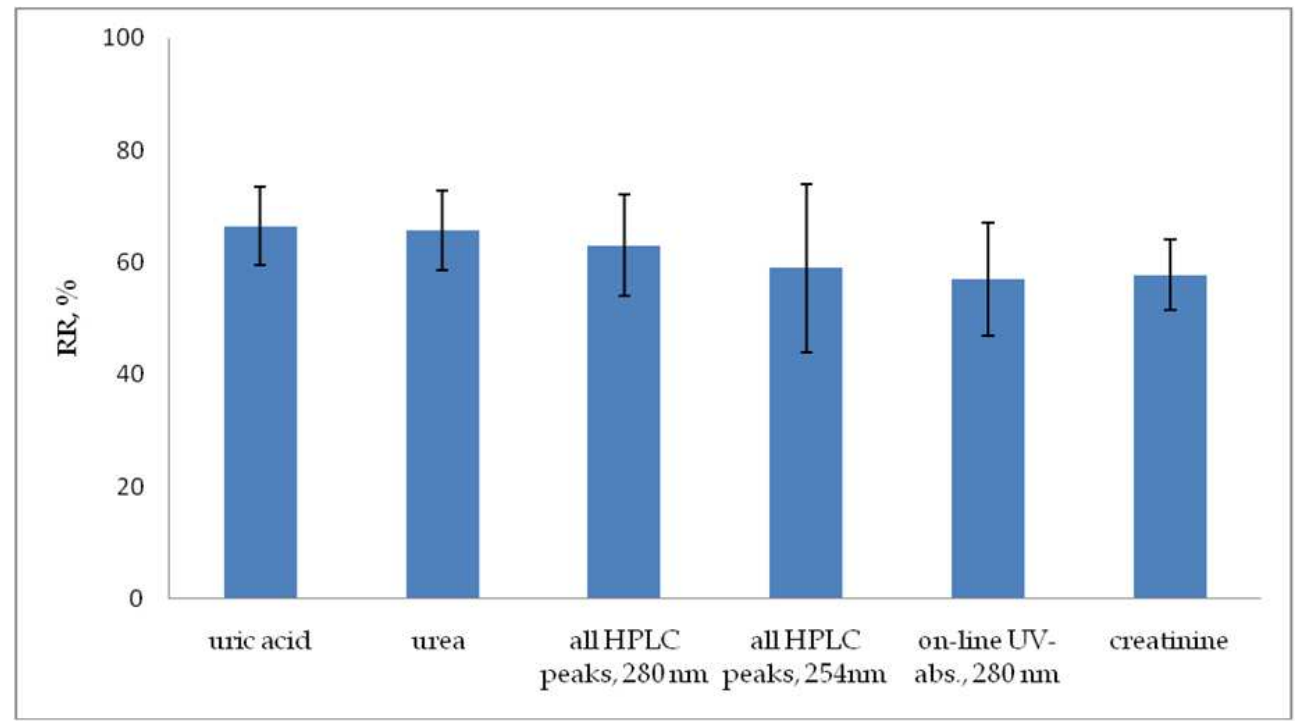

Fig. 8. Comparison of RR of small molecular uremic retention solutes (uric acid, urea and creatinine) in serum, RR of all HPLC serum peaks on two different wavelengths ( 254 and $280 \mathrm{~nm})$ and spent dialysate online UV absorbance $(280 \mathrm{~nm})$ 
The RR of urea and uric acid and the RR of all HPLC peaks at $280 \mathrm{~nm}$ are $>60 \%$, while the RR of all HPLC peaks at $254 \mathrm{~nm}$, of online UV absorbance measurement and creatinine are < $60 \%$. Interestingly, the removal of urea and uric acid was statistically undifferentiated $(\mathrm{p}>$ $0.05)$. Figure 8 shows that the RR of serum creatinine is statistically different $(p<0.05)$ from the RR of serum urea and uric acid concentrations and from the RR of all HPLC peaks in the serum (at $280 \mathrm{~nm}$ ), but not different from that of $254 \mathrm{~nm}$. The RR of online measurement is comparable with the RR of creatinine and of all HPLC peaks at $254 \mathrm{~nm}$ and lower than the RR of urea, uric acid and all HPLC peaks at $280 \mathrm{~nm}(\mathrm{p}<0.05)$. At the same time, the RR of serum urea and uric acid are not statistically different; neither are they different from the RR of all HPLC peaks at $254 \mathrm{~nm}$ and $280 \mathrm{~nm}(\mathrm{p}<0.05)$.

The correlation coefficients between RR UV absorbance at $254 \mathrm{~nm}$ and at $280 \mathrm{~nm}$ from the total area of the HPLC peaks, from online monitoring at $280 \mathrm{~nm}$ and RR for certain substances with different molecular weights in spent dialysate and serum are shown in Table 2.

\begin{tabular}{|l|c|c|c|}
\hline & Uric acid & Urea & Creatinine \\
\hline RR of all HPLC peaks, $254 \mathrm{~nm}$ & 0.755 & 0.811 & 0.812 \\
\hline RR of all HPLC peaks, $280 \mathrm{~nm}$ & 0.933 & 0.881 & 0.926 \\
\hline Online UV absorbance, $280 \mathrm{~nm}$ & 0.890 & 0.873 & 0.888 \\
\hline
\end{tabular}

Table 2. Pearson correlation coefficients between RR of all HPLC peaks ( $254 \mathrm{~nm}$ and 280 $\mathrm{nm}$ ), online absorbance at $280 \mathrm{~nm}$ and RR of uric acid, urea and creatinine in serum. The significance level of the results is $\mathrm{P}<0.01$.

A high correlation for uric acid, urea and creatinine was obtained in both spent dialysate and serum. Some differences regarding $254 \mathrm{~nm}$ and $280 \mathrm{~nm}$ can be observed. However, urea does not represent as good a correlation as uric acid.

\section{Discussion}

From the investigations presented in this chapter we can conclude the following: (i) UV absorbance decreases during the dialysis session as the waste products are removed; (ii) a number of higher prevalent peaks can be detected in the HPLC profiles; (iii) some lowmolecular-weight uremic solutes were identified from the HPLC profiles contributing to UV absorbance, and the main solute responsible for the UV absorbance is uric acid; (iv) a difference between HPLC profiles on wavelengths of 254 and $280 \mathrm{~nm}$ was found; (v) a higher number of detected HPLC peaks in the serum comparing to spent dialysate has been detected; (vi) the low flux and high flux membranes showed similar RR for all studied uremic solutes; and (vii) the classic dialysis adequacy marker urea and the uremic solute uric acid have good correlation with uremic retention solute elimination forming the UV absorbance curve.

Figures 2 and 5 show how the concentration of the uremic retention solutes - chromophores - decreases during a dialysis session in spent dialysate. UV absorbance as well as the height of the HPLC peaks are higher at the beginning of the treatment because of the high concentration of metabolic waste products in the body fluids and UV absorbance is lower at the end of the dialysis session. This demonstrates the possibility of following a single dialysis session continuously and to monitor deviations during treatment using a UV absorbance online monitor. This enables us to estimate the dialysis efficiency and adjust the treatment settings if needed. 
A number of higher prevalent HPLC peaks representing chromophores can be observed (Figure 5 and 6). This indicates that there is a group of compounds, among them several uremic toxins, which are the main cause of cumulative and integrated UV absorbance. The 10 main peaks formed app. $80-90 \%$ of the total area of all HPLC peaks; some of these are small molecular weight uremic toxins such as uric acid, creatinine, hippuric acid and indoxyl sulphate. The variations in the number of HPLC peaks depending on hemodialysis treatments and patients have been demonstrated in earlier studies (Schoots, 1982; Vanholder, 1992). The difference between two dialysis sessions may arise as shown in Figure 2, because of the different composition and removal of the uremic retention solutes contained in spent dialysate. When comparing the HPLC profiles of the spent dialysate in Figure 5 and those of the serum in Figure 6, more peaks are detected in the serum. Thus, not all solutes in serum are transported to dialysate and removed through the semi-permeable membrane.

The number of detected HPLC peaks at $254 \mathrm{~nm}$ and $280 \mathrm{~nm}$ is also demonstrated in Figure 6 and 7. The difference in the number of detected HPLC peaks on the wavelengths of $254 \mathrm{~nm}$ and $280 \mathrm{~nm}$ arises due to the characteristic absorbing spectra of the UV chromophores. The absorption of many components is higher on the wavelength of $254 \mathrm{~nm}$ than $280 \mathrm{~nm}$. This confirms the results obtained via the spectrophotometric analysis in this UV region (Fridolin, 2003). However, the studies of relations between UV and small water soluble molecules such as urea and uric acid indicated that the wavelength of $280 \mathrm{~nm}$ may be preferred for online measurements when small water soluble molecules should be estimated. On this wavelength a relatively strong linear relationship exists between UV absorbance and concentrations of urea, creatinine and uric acid (Fridolin, 2002; Uhlin, 2003). While the contribution of uric acid forms a considerable part of the total area of HPLC peaks, uric acid plays an important role in online UV absorbance dialysis dose monitoring. Interestingly, the removal of urea and uric acid was statistically undifferentiated ( $p>0.05)$. This information gives us alternative possibilities to use other components and methods to monitor urea reduction (URR) in a single hemodialysis session.

Additionally, the low flux and high flux membranes showed no different removal of the studied small molecule uremic toxins as presented in earlier studies (Lesaffer, 2000). In this study it was found that the cellulose triacetate and polysulphone HF membranes removed similarly classical markers and protein-bound liphophilic solutes as an LF polysulphone membrane. Parallel results were obtained even with the concentrations corrected using a correction factor based on the total protein concentration at the start and at the end of dialysis as used by Lesaffer et al. (Lesaffer, 2000). Furthermore, there was no statistical difference between intradialytic start-end values, and removal efficiency for the LF and HF membranes estimated by the total area of HPLC peaks at $254 \mathrm{~nm}$ and $280 \mathrm{~nm}$ in the serum and online UV absorbance at $280 \mathrm{~nm}$ in the spent dialysate. This indicates that UV absorbance follows the behaviour of UV-absorbing compounds - uremic toxins - which are the origin of total UV absorbance in serum and spent dialysate.

The RR values of different identified compounds, the total area of all HPLC UV absorbance peaks on the wavelengths of $254 \mathrm{~nm}$ and $280 \mathrm{~nm}$ in the serum and the RR of online UV absorbance at $280 \mathrm{~nm}$ in spent dialysate are presented in Table 1 and Figure 8. Taking into account the removal efficiency, a difference can be observed in the relation of UV absorbance to small water-soluble non-protein-bound solutes and to small protein-bound solutes such as indoxyl sulphate. The small non-protein-bound solutes uric acid and urea showed a far more substantial decrease of concentration than creatinine being statistically 
different $(p<0.05)$ from the RR of serum uric acid and urea. The similar removal of urea and uric acid makes it possible to use other components and methods to monitor urea reduction during a single hemodialysis session. At the same time, the RR of creatinine is statistically different ( $p<0.05)$ from the RR of all HPLC peaks in the serum at $280 \mathrm{~nm}$, but not different from that of $254 \mathrm{~nm}$. The RR for online UV absorbance is lower compared to urea. Considering that RR (URR) is correlated to Kt/V (NKF-DOQI, 2006), this tendency is reported earlier, as the dialysis dose estimated by online UV absorbance was lower than $\mathrm{Kt} / \mathrm{V}$ urea (Uhlin, 2003). The difference between the RR of all HPLC peaks in serum and online UV absorbance measurement (at $280 \mathrm{~nm}$ ) in spent dialysate could be due to different chromophores in the serum and spent dialysate and because the serum was collected before and the dialysate sample 10 minutes after the start of dialysis. Moreover, the different binding of individual uremic retention solutes to serum proteins may modify percentage concentration changes of individual solutes in the course of haemodialytic treatment (Vanholder, 1992), supported by observations of decreased drug/protein interactions in uremic serum (De Smet, 1999).

The correlation analysis also provides additional insights into the removal characteristics of solutes and UV absorbance monitoring (Table 2). The RR of uric acid has the highest correlation for RR at $280 \mathrm{~nm}$ in both serum and spent dialysate, but not at $254 \mathrm{~nm}$ in serum. The explanation is the highest contribution of uric acid to UV absorbance compared to other chromophores at $280 \mathrm{~nm}$ (Figures 5 and 6). However, there are several other strong contributions from other compounds beside uric acid at $254 \mathrm{~nm}$, and therefore the correlation is lower. The outcome in Table 2 is confirmed by comparing the millimolar extinction coefficients versus wavelengths for uric acid (Vasilevsky, 2005). A higher value of the extinction coefficient corresponds to the higher correlation for RR of uric acid at $280 \mathrm{~nm}$.

The RR of urea is more related to RR at $280 \mathrm{~nm}$ both in serum and spent dialysate, but less so at $254 \mathrm{~nm}$ in serum (Fridolin, 2003). This means that relatively good correlation between the RR of UV absorbance and a particular solute may be achieved when the removal rate of a non-absorbing solute (e.g. urea) is similar to that of UV-absorbing substances during haemodialysis. This is also confirmed by very good correlation between several small molecular weight waste products and UV absorbance (Fridolin, 2002) and similar concentration changes during dialysis for several azotemic markers (e.g. urea, creatinine and uric acid) (Vanholder, 1992). The dominance of small molecular weight waste products among chromophores in serum and spent dialysate can be concluded because the number of detected HPLC peaks is not significantly different for serum filtered with a filter in cut-off 3 $\mathrm{kDa}$ and $70 \mathrm{kDa}$. Furthermore, it seems that the UV-absorbing solutes can be subject to similar corrections regarding distribution volume and intercompartmental equilibration rates, similar to urea, although not with exactly the same distribution and equilibration intercompartmental rates in the body as urea. This makes it possible to estimate the delivered dialysis dose in terms of $\mathrm{Kt} / \mathrm{V}$ by monitoring $\mathrm{UV}$ absorbance in spent dialysate online (Uhlin, 2003).

The RR of creatinine demonstrates a high correlation for RR at $280 \mathrm{~nm}$ in both serum and spent dialysate. The reason could be similar removal of creatinine and other chromophores at $280 \mathrm{~nm}$, where creatinine does not contribute significantly to UV absorbance (Figure 5).

As previous studies have shown, and as is confirmed by this work, the HPLC is a method which has its own place for the detection of uremic solutes in biological solutes. This is an effective method of studying accumulated metabolites in patients' blood and removed in dialysis. Identification of these metabolites gives us the opportunity to understand the 
cumulative and integrated UV absorbance curve utilised during optical dialysis dose online monitoring. The online methods are felt to be more accurate than methods based on preand post-dialysis urea concentrations, and to be better suited to clinical routine. Continuous monitoring of uremic retention solute concentrations during a dialysis session could be beneficial for the prevention of intradialytic morbidity and for the confirmation of dialysis adequacy.

\section{Conclusions}

This chapter contributes new information about the removal of uremic retention solutes during hemodialysis and the origin of the optical dialysis adequacy monitoring signal. The relationship between characteristics of the online UV absorbance curve measured during dialysis and the identified HPLC peaks in spent dialysate was investigated. It was demonstrated that the absorbance signal reflects the contribution of several UV-absorbing compounds in spent dialysate, with the strongest influence coming from the low-molecularweight water-soluble non-protein bound compounds. Moreover, UV absorbance behaves more like small water-soluble non-protein-bound solutes than small protein-bound solutes.

Monitoring the removal of compounds with different properties and elimination characteristics during various dialysis strategies adds knowledge of dialysis treatment and would be useful for future research in order to decrease complications related to dialysis quality and cardiovascular risk factors. Hopefully the online methods will add a new technique and methodology to the wide discussion about the quality and adequacy of dialysis, uremic toxicity and kidney functionality.

\section{Acknowledgements}

The authors wish to thank the nurses and technical staff who assisted us during our clinical experiments as well as the dialysis patients who kindly participated in the experiments. This study was supported by the Estonian Science Foundation (grant no. 8621), the Estonian targeted financing project SF0140027s07 and the European Union through the European Regional Development Fund.

\section{References}

Barnett, A.L. \& Veening, H. (1985) Liquid-chromatographic study of fluorescent compounds in haemodialysate solutions, Clinical Chemistry, Jan.31(1), pp. 127-130

Castellarnau, A., Werner, M., Günthner, R. \& Jakob, M. (2010). Real-time Kt/V determination by ultraviolet absorbance in spent dialysate: technique validation, Kidney International, 78, November (1), pp. 920-925

Cho, D. S., Olesberg, J.T., Flanigan, M.J. \& Arnold, M. (2008). Online Near-Infrared Spectrometer to Monitor Urea Removal in Real Time During Hemodialysis, Applied Spectroscopy, Vol. 62, Issue 8, pp. 866-872

De Smet, R., Vogeleere, P. et al. (1999). Study by means of high-performance liquid chromatography of solutes that decrease theophylline protein binding in the serum of uremic patients, Journal of Chromatography A., 847(1-2), pp. 141-153 
De Smet, R., Dhondt, A., Galli, F., Waterloos, M.A. \& Vanholder, R. (2007). Effect of the super-flux cellulose triacetate dialyser membrane on the removal of non-proteinbound and protein-bound uraemic solutes, Nephrol Dial Transplant, 22, pp. 20062012

Eddy, C.M. \& Arnold, M.A. (2001). Near-infrared spectroscopy for measuring urea in hemodialysis fluids, Clin Chem., 47 (7), pp. 1279-1286

Eddy, C. V., Flanigan, M. et al. (2003). Near-Infrared Spectroscopic Measurement of Urea in Dialysate Samples Collected During Hemodialysis Treatments, Applied Spectroscopy, 57(10), pp. 1230-6

Eloot, S., Torremans, A., De Smet, R., Marescau, B., De Deyn, PP., Verdonck, P. \& Vanholder, R. (2007). Complex compartmental behavior of small water-soluble uremic retention solutes: evaluation by direct measurements in plasma and erythrocytes, Am J Kidney Dis., 50, pp. 279-288

Eloot, S., Van Biesen, W., Dhondt, A., Van de Wynkele, H., Glorieux, G., Verdonck, P. \& Vanholder, R. (2008). Impact of hemodialysis duration on the removal of uremic retention solutes, Kidney International, 73, pp. 765-770

Eknoyan, G., Beck, G.J., Cheung, A.K., et al. (2002). Effect of dialysis dose and membrane flux in maintenance hemodialysis, N Engl J Med, 347, pp. 2010-2019

Ficheux, A., Gayrard, N., et al. (2010). The use of SDS-PAGE scanning of spent dialysate to assess uraemic toxin removal by dialysis, Nephrol Dial Transplan, Dec 10

Fridolin, I., Magnusson, M. \& Lindberg, L.-G. (2002). Online monitoring of solutes in dialysate using absorption of ultraviolet radiation: technique description, Int J Artif Organs, 25, pp. 748-761

Fridolin, I. \& Lindberg, L.G. (2003). Online monitoring of solutes in dialysate using absorption of ultraviolet radiation - wavelength dependence, Medical $\mathcal{E}$ Biological Engineering \& Computing, 41, pp. 263-270

Jensen, P. S. \& Bak, J. (2002). Measurements of urea and glucose in aqueous solutions with dual-beam near-infrared Fourier transform spectroscopy, Applied Spectroscopy, 56(12), pp. 1593-1599

Kaplan, B., Cojocaru, M., Unsworth, E., Knecht, A. \& Martin, B.M. (2003). Search for peptidic "middle molecules" in uremic sera: Isolation and chemical identification of fibrinogen fragments, J Chromatogr B., 796, pp. 141-153

Knudson, E.J., Lau, Y.C., Veening, H. \& Dayton, D.A. (1978). Time-concentration studies by high-performance liquid chromatography of metabolites removed during hemodialysis, Clinical Chemistry, Apr. 24(4), pp.686-91

Lauri, K., Tanner, R., Jerotskaja, J., Luman, M. \& Fridolin, I. (2010). HPLC study of uremic fluids related to optical dialysis adequacy monitoring, Int J Artif Organs, 33 (2), pp. 96-104

Lesaffer, G., De Smet, R., Lameire, N., Dhondt, A., Duym, P. \& Vanholder, R. (2000). Intradialytic removal of protein-bound uraemic toxins: role of solute characteristics and of dialyser membrane, Nephrol Dial Transplant, 15, pp. 50-57

Locatelli, F., Buoneristiani, U., Canaud, B., Khler, H., Petitclerc, T. \& Zucchelli, P. (2005). Hemodialysis with online monitoring equipment: tools or toys?, Nephrol Dial Transplant, 20, pp. 22-33 
Niwa, T. (1993). Phenol and p-cresol accumulated in uremic serum measured by HPLC with fluorescence detection, Clinical Chemistry, Vol 39, pp. 108-111

Niwa, T. (1997). Mass spectrometry in the search for uremic toxins. Mass Spectrom Rev,. NovDec,16(6), pp. 307-32

Niwa, T. (2009). Recent progress in the analysis of uremic toxins by mass spectrometry, J Chromatogr B Analyt Technol Biomed Life Sci., Sep 1, 877(25), pp. 2600-2606

Niwa, T. (2011). Update of uremic toxin research by mass spectrometry, Mass Spectrometry Reviews, 30 (3), pp. 510-521

NKF-DOQI (2006). Clinical practice guidelines for hemodialysis adequacy, Am J Kidney Dis., 48, pp. 13-97

Olesberg, J.T., Arnold, M.A., et al. (2004). Online Measurement of Urea Concentration in Spent Dialysate during Haemodialysis,. Clin Chem., 50(1), pp. 175-81

Schoots, A.C., Mikkers, F.E.P., et al. (1982). Characterization of Uremic "Middle Molecular" Fractions by Gas Chromatography, Mass Spectrometry, Isotachophoresis, and Liquid Chromatography, Clin Chem., 28/1, pp. 45-49

Schoots, Ad.C., Homan, H.R., Gladdines, M.M, Cramers, C.A.M.G, De Smet, R. \& Ringoir Severin, M. G. (1985). Screening of UV-absorbing solutes in uremic serum by reversed phase HPLC - Change of blood levels in different therapies, Clinica Chimica Acta, Volume 146, Issue 1, 28 February, pp. 37-51

Schoots, A.C, Peeters, J.A. \& Gerlag, P.G. (1989). Effect of hemodialysis on serum concentrations of HPLC-analyzed accumulating solutes in uremia, Nephron, 53(3), pp. 208-217

Senftleber, F., Halline, A.G., Veenlng, H. \& Dayton, D.A. (1976). Reversed-Phase LiquidChromatographic Analysis of Haemodialysate from Uremic Patients, Clinical Chemistry, 22 (9), pp. 1522-1527

Stiller, S., Al-Bashir A. \& Mann H. (2001). Online Urea Monitoring during Hemodialysis: A Review, Saudi Journal of Kidney Diseases and Transplantation, 12 (3), pp-364-374

Swan, J.S., Kragten, E.Y. \& Veening, H. (1983). Liquid-chromatographic study of fluorescent materials in uremic fluids, Clinical Chemistry, Jul, 29(6), pp. 1082-1084

Uhlin, F., Fridolin, I., Lindberg, L.-G. \& Magnusson, M. (2003). Estimation of delivered dialysis dose by online monitoring of the UV absorbance in the spent dialysate, Am J Kidney Dis, 41, pp. 1026-1036

Vanholder, R., De Smet, R.V. \& Ringoir, S.M. (1992). Assessment of urea and other uremic markers for quantification of dialysis efficacy, Clin Chem, vol. 38, pp. 1429-1436

Vasilevsky, A.M. \& Konoplyov, G.A.. (2005). Peculiar character of dialysate ultraviolet extinction spectra as an indicator of nucleic acid metabolism in humans, Journal of Biomedical Optics, 10(4), pp. 044026/1-7

Vanholder, R., De Smet, R. \& Lameire, N.H. (2001). Redesigning the map of uremic toxins, Contr. Nephrol., 133, pp. 42-70

Vanholder R., De Smet R., et al. (2003). Survival of Hemodialysis Patients and Uremic Toxin Removal, Artificial Organs, 27(3), pp. 218-223

Vanholder, R. et al. (2008). A Bench to Bedside View of Uremic Toxins, J Am Soc Nephrol, 19, pp. $863-870$ 
Vanholder, R., Laecke, S. V., Verbeke, F., Glorieux, G. \& Biesen, W.V. (2008). Uraemic toxins and cardiovascular disease: in vitro research versus clinical outcome studies, NDT Plus, 1, pp. 2-10

Vanholder, R. (2009). Effect of membrane permeability on survival of hemodialysis patients, J Am Soc Nephrol, 20, pp. 645-654 


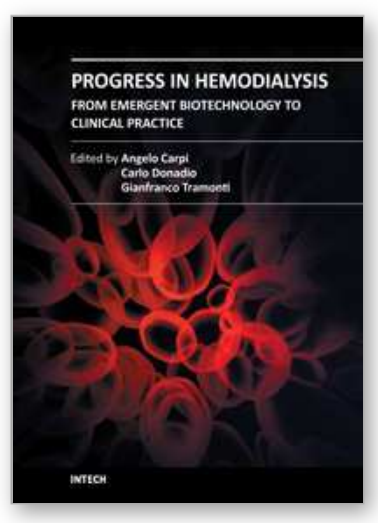

\section{Progress in Hemodialysis - From Emergent Biotechnology to Clinical Practice}

Edited by Prof. Angelo Carpi

ISBN 978-953-307-377-4

Hard cover, 444 pages

Publisher InTech

Published online 07, November, 2011

Published in print edition November, 2011

Hemodialysis (HD) represents the first successful long-term substitutive therapy with an artificial organ for severe failure of a vital organ. Because HD was started many decades ago, a book on HD may not appear to be up-to-date. Indeed, HD covers many basic and clinical aspects and this book reflects the rapid expansion of new and controversial aspects either in the biotechnological or in the clinical field. This book revises new technologies and therapeutic options to improve dialysis treatment of uremic patients. This book consists of three parts: modeling, methods and technique, prognosis and complications.

\section{How to reference}

In order to correctly reference this scholarly work, feel free to copy and paste the following:

Kai Lauri, Jürgen Arund, Jana Holmar, Risto Tanner, Merike Luman and Ivo Fridolin (2011). Optical Dialysis Adequacy Monitoring: Small Uremic Toxins and Contribution to UV-Absorbance Studied by HPLC, Progress in Hemodialysis - From Emergent Biotechnology to Clinical Practice, Prof. Angelo Carpi (Ed.), ISBN: 978-953307-377-4, InTech, Available from: http://www.intechopen.com/books/progress-in-hemodialysis-fromemergent-biotechnology-to-clinical-practice/optical-dialysis-adequacy-monitoring-small-uremic-toxins-andcontribution-to-uv-absorbance-studied-b

\section{INTECH}

open science | open minds

\section{InTech Europe}

University Campus STeP Ri

Slavka Krautzeka 83/A

51000 Rijeka, Croatia

Phone: +385 (51) 770447

Fax: +385 (51) 686166

www.intechopen.com

\section{InTech China}

Unit 405, Office Block, Hotel Equatorial Shanghai

No.65, Yan An Road (West), Shanghai, 200040, China

中国上海市延安西路65号上海国际贵都大饭店办公楼 405 单元

Phone: +86-21-62489820

Fax: $+86-21-62489821$ 
(C) 2011 The Author(s). Licensee IntechOpen. This is an open access article distributed under the terms of the Creative Commons Attribution 3.0 License, which permits unrestricted use, distribution, and reproduction in any medium, provided the original work is properly cited. 